\title{
GLUON CONTRIBUTIONS TO SMALL $x$ HEAVY FLAVOUR PRODUCTION
}

\author{
S. CATANI ${ }^{\mathrm{a}, \mathrm{b}}$, M. CIAFALONI ${ }^{\mathrm{c}, \mathrm{a}}$ and F. HAUTMANN ${ }^{\mathrm{c}, \mathrm{a}}$ \\ a Istituto Nazionale di Fisica Nucleare, Sezione di Firenze, I-50125 Florence, Italy \\ - Cavendish Laboratory, University of Cambridge, Cambridge CB3 OHE, UK \\ c Dipartimento di Fisica, Universitá di Firenze, 1-50125 Florence, Italy
}

Received 15 March 1990

\begin{abstract}
We show that the large QCD corrections to heavy flavour production due to small $x$ logarithms can be evaluated and resummed in closed form, including the coefficient factor. The argument is based on a $k_{1}$-dependent factorization of the Born and structure function terms which is ascribed to Regge behaviour. The photoproduction case is investigated in detail. At high energies the total cross section turns out to be much larger than that obtained by a naive renormalization group approach.
\end{abstract}

Heavy flavour production in the present $\mathrm{TeV}$ range, or at higher energies, is characterized by small values of the parameters $\rho=4 \mathrm{M}^{2} / \mathrm{s}$ in both lepton- and hadron-initiated processes. In quantum chromodynamics, such two-scale processes [1] show large perturbation terms which are $\mathrm{O}(\log (1 / \rho))$ (if $s \gg M^{2}$ $\gg \Lambda^{2}$ ), in addition to the usual ones - predicted by the renormalization group - which are $\mathrm{O}\left(\log \left(M^{2}\right)\right.$ $\left.\Lambda^{2}\right)$ ).

In low order calculations [2-5], the constant or logarithmic terms just mentioned affect, for $\rho \rightarrow 0$, both the anomalous dimension and the coefficient factor. For instance, in the photoproduction case, the first order QCD corrections to the Born cross section, with one associated gluon, is given by [3]

$\sigma_{\mathrm{gg}}^{(1)}\left(M^{2}, \rho\right)=\frac{\alpha}{M^{2}} \alpha_{\mathrm{s}}^{2}\left(M^{2}\right)\left(c_{1}(\rho)+\bar{c}_{\mathrm{I}}(\rho) \log \frac{M^{2}}{Q_{0}^{2}}\right)$,

where, for large energy or small $\rho$, one has the constant limits

$c_{1} \rightarrow \frac{41}{9} e_{\mathrm{Q}}^{2}, \quad \bar{c}_{1} \rightarrow \frac{7}{3} e_{\mathrm{Q}}^{2}$.

Here $\alpha_{\mathrm{s}}$ and $\Lambda$ are the QCD coupling constant and scale, $Q_{0}$ is a factorization scale ( $\operatorname{such}$ as $\alpha_{\mathrm{s}}\left(Q_{0}^{2}\right) \leqslant 1$ ), and $e_{\mathrm{Q}}$ is the heavy flavour charge.

\footnotetext{
* Research supported in part by the UK Science and Engineering Research Council.
}

The constant behaviour in (2) is due to gluon exchange (fig. 1) and will generate, at higher orders, powers of $\log (1 / \rho)$ which are not necessarily associated with powers of $\log \left(M^{2} / Q_{0}^{2}\right)$, thus affecting also the coefficient factor. While the anomalous dimension terms are expected to be universal and described, in moment space, by Lipatov's singularity [6], the coefficient terms are process dependent, and do not seem to follow any simple rule.

The purpose of this note is to show that actually all leading logs of the coefficient function can be evaluated and resummed.

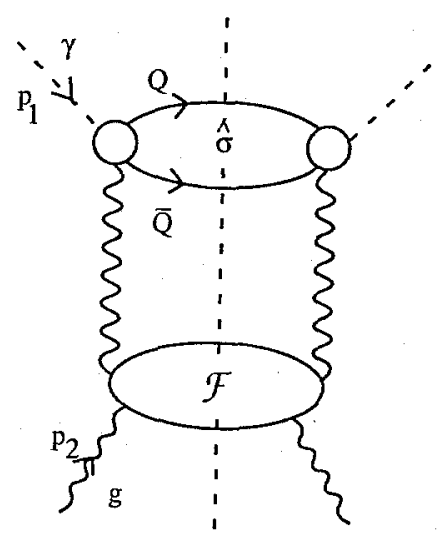

Fig. 1. Factorized structure of the heavy flavour photoproduction cross section in the high energy limit. 
The basic observation that we make is that in leading logarithmic approximation, the photoproduction cross section satisfies a $k_{\perp}$-dependent factorization formula, as follows (fig. 1):

$$
\begin{aligned}
& 4 M^{2} \sigma\left(\rho, \frac{M^{2}}{Q_{0}^{2}}\right) \\
& =\int \mathrm{d}^{2} \boldsymbol{k} \int_{0}^{1} \frac{\mathrm{d} z}{z} \hat{\sigma}\left(\frac{\rho}{z}, \frac{\boldsymbol{k}^{2}}{4 M^{2}}\right) \mathscr{F}\left(z, \boldsymbol{k} ; Q_{0}^{2}\right) .
\end{aligned}
$$

Here $\mathscr{F}(x, \boldsymbol{k})$ is the unintegrated gluon structure function (defined below) and $\hat{\sigma}$ is the off-shell $\gamma+\mathrm{g}(k) \rightarrow \mathrm{Q}+\overline{\mathrm{Q}}$ Born cross section, defined by coupling the external gluons to high energy partons with eikonal vertices (fig. 2 ).

More precisely, if $A^{\mu \nu}\left(p_{1} ; k\right)$ is the $\gamma \mathrm{g}$ absorptive part at lowest order (fig. $2 a$ ), we define

$\hat{\sigma}\left(\frac{4 M^{2}}{s}, \frac{\boldsymbol{k}^{2}}{4 M^{2}}\right)=\frac{4 M^{2}}{s} \frac{p_{2}^{\mu} p_{2}^{\nu}}{\boldsymbol{k}^{2}} A_{\mu \nu}\left(p_{1} ; k\right)$,

$s=2 p_{1} \cdot p_{2}, \quad p_{1}^{2}=p_{2}^{2}=0$,

while $\mathscr{F}(x, \boldsymbol{k})$ is assumed to satisfy the Lipatov equation [6] for gluons (fig. 2b), in the form of ref. [1], i.e. $\left(\bar{\alpha}_{\mathrm{s}} \equiv N_{\mathrm{c}} \alpha_{\mathrm{s}} / \pi\right)$,

$$
\begin{aligned}
& \mathscr{F}(x, \boldsymbol{k})=\delta(x-1) \delta^{(2)}(\boldsymbol{k}) \\
& +\bar{\alpha}_{\mathbf{s}} \int \frac{\mathrm{d}^{2} \boldsymbol{q}}{\pi \boldsymbol{q}^{2}} \frac{\mathrm{d} z}{z}\left[\mathscr{F}\left(\frac{x}{z}, \boldsymbol{k}+\boldsymbol{q}\right)\right. \\
& \left.-\Theta\left(\boldsymbol{k}^{2}-\boldsymbol{q}^{2}\right) \mathscr{F}\left(\frac{x}{z}, \boldsymbol{k}\right)\right] .
\end{aligned}
$$

Leaving to later work [7] a formal proof of eq. (3), we note that its factorization structure is $k_{\perp}$-dependent, thus generalizing the usual renormalization group statement. Its basis is in the high-energy, or Regge-pole, factorization for exchange gluons. In fact, if both $s / M^{2}$ and $M^{2}$ are large, the single gluon exchange diagram of fig. 1 is dominant, in a physical gauge, for any fixed $k^{2} \simeq-k^{2}$. High energy factorization then implies that the gluon structure function factors out as function of $k$, and that the Born factor $\hat{\sigma}$ is defined as a physical cross section for the high energy process in fig. 2a. Therefore eq. (3) holds, and $\hat{\sigma}$ is gauge invariant and calculable (at leading $s$ level) despite its off-shell $k_{\perp}$-dependence.

All features of the large perturbation terms follow from eq. (3). Note first that since $\hat{\sigma}$ itself is small for $\rho \rightarrow 0[\hat{\sigma} \sim \rho \log (1 / \rho)]^{\# 1}$, the constant or log terms mentioned before are generated by the singular $z$ integration over the gluon fragmentation densities in

\#1 This feature is due to the fermion exchange diagrams involved in the Born cross section.

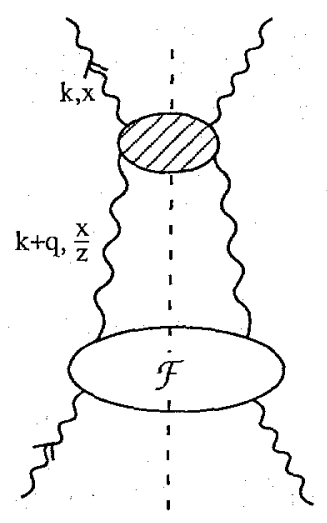

(b)

Fig. 2. (a) One-loop correction to the Born photoproduction cross section in the Regge limit, and (b) iteration kernel for the Lipatov equation. 
$\mathscr{F}$. Furthermore, the small $k_{\perp}$ behaviour of the structure function $\left(\mathscr{F} \sim\left(\boldsymbol{k}^{2}\right)^{-1}\right.$ for $\left.A \ll k_{\perp} \ll M\right)$ is responsible for the anomalous dimension terms, while large $k_{\perp}$ 's are cut-off by $\hat{\sigma}$ at $k_{\perp} \sim M$ and contribute to the coefficient factor. Finally, since the small $z$ behaviour of $\mathscr{F}$ is known to all orders from eq. (5), eq. (3) can be used to sum all leading logs in the heavy flavour cross section.

The detailed analysis of the high energy limit $(\rho \rightarrow 0)$ of the expression (3) requires the explicit form of the differential Born cross section. By introducing the angular yariable

$\xi=4 \alpha(1-\alpha), \quad \alpha=\frac{M^{2}-\hat{t}}{z s}$,

and the phase-space factor

$\mathrm{d} \Phi=\frac{1}{16 \pi} \frac{\mathrm{d} \xi}{\sqrt{1-\xi}} \Theta\left(\frac{z}{\rho}-\frac{1}{\xi}-\frac{\boldsymbol{k}^{2}}{4 M^{2}}\right)$,

we obtain [7], from the definition (4),

$\mathrm{d} \hat{\sigma}\left(\frac{\rho}{z}, \xi, \frac{\boldsymbol{k}^{2}}{4 M^{2}}\right)=\frac{\rho}{z} A\left(\frac{\rho}{z}, \xi, \frac{\boldsymbol{k}^{2}}{4 M^{2}}\right) \mathrm{d} \Phi$,

where the spin-averaged absorptive part $A$ is given by a second order polynomial in $\boldsymbol{k}^{2}$ as follows:

$$
\begin{aligned}
& A\left(\rho, \xi, \frac{\boldsymbol{k}^{2}}{4 M^{2}}\right) \\
& \quad=32 \pi^{2} \alpha \alpha_{\mathrm{s}} e_{\mathrm{Q}}^{2} \frac{1}{\xi} \sum_{n=0}^{2} a_{n}(\rho, \xi)\left(\frac{\boldsymbol{k}^{2}}{4 M^{2}}\right)^{n}, \\
& a_{0}=1-\frac{\xi}{2}+\rho-\frac{\rho^{2}}{\xi}, \quad a_{1}=\rho\left(3 \xi-2-4 \rho+\frac{2 \rho}{\xi}\right), \\
& a_{2}=\rho^{2}(2-3 \xi) .
\end{aligned}
$$

Note now that eq. (3) is a convolution in $\rho$-space with $k_{\perp}$-factorization. Thus, its $\rho$-moments can be written as

$$
\begin{aligned}
& 4 M^{2} \sigma_{N}\left(\frac{M^{2}}{Q_{0}^{2}}\right)=\int \mathrm{d}^{2} \boldsymbol{k} \mathscr{F}_{N}\left(\boldsymbol{k} ; Q_{0}^{2}\right) \hat{\sigma}_{N}\left(\frac{\boldsymbol{k}^{2}}{4 M^{2}}\right) \\
& =\int_{1 / 2-\mathrm{i} \infty}^{1 / 2+\mathrm{i} \infty} \frac{\mathrm{d} \gamma}{2 \pi \mathrm{i}}\left(\frac{M^{2}}{Q_{0}^{2}}\right)^{\gamma}\left(1-\frac{\bar{\alpha}_{\mathrm{s}}}{N} f(\gamma)\right)^{-1} \frac{1}{\gamma} h_{N}(\gamma),
\end{aligned}
$$

where we have expressed the solution $\mathscr{F}_{N}(\boldsymbol{k})$ of eq. (5) in the form

$$
\begin{aligned}
& \mathscr{F}_{N}\left(\boldsymbol{k} ; Q_{0}^{2}\right) \\
& \quad=\frac{1}{\pi \boldsymbol{k}^{2}} \int_{1 / 2-\mathrm{i} \infty}^{1 / 2+\mathrm{i} \infty} \frac{\mathrm{d} \gamma}{2 \pi \mathrm{i}}\left(\frac{\boldsymbol{k}^{2}}{Q_{0}^{2}}\right)^{\gamma}\left(1-\frac{\overline{\alpha_{\mathrm{s}}}}{N} f(\gamma)\right)^{-1}, \\
& f(\gamma)=2 \psi(1)-\psi(\gamma)-\psi(1-\gamma),
\end{aligned}
$$

$f(\gamma)$ being the well-known [1] Lipatov anomalous dimension function given in terms of Euler $\psi$-functions. The Born cross section enters eq. (9) through the weighted $k_{\perp}$-average $h_{N}$, defined by

$$
\frac{1}{\gamma} h_{N}(\gamma)=\int_{0}^{\infty} \frac{\mathrm{d} \boldsymbol{k}^{2}}{\boldsymbol{k}^{2}}\left(\frac{\boldsymbol{k}^{2}}{M^{2}}\right)^{\gamma} \hat{\sigma}_{N}\left(\frac{\boldsymbol{k}^{2}}{4 M^{2}}\right) \text {. }
$$

Since the differential cross section in eqs. (7) and (8) is vanishingly small for $\rho \rightarrow 0$ and any $k_{\perp}$ (small or large, i.e. $\left.k_{\perp}^{2} \sim M^{2} / \rho\right), h_{N}$ is weakly $N$-dependent, i.e. $h_{N}=h(\gamma)[1+\mathrm{O}(N)]$. A straightforward calculation yields

$$
\begin{aligned}
& h(\gamma)=\frac{4 \pi}{3} \alpha \alpha_{\mathrm{s}} e_{\mathrm{Q}}^{2} \frac{7-5 \gamma}{3-2 \gamma} \\
& \quad \times B(1-\gamma, 1-\gamma) B(1+\gamma, 1-\gamma),
\end{aligned}
$$

where $B$ is Euler's beta function. Note the finite value of $h(0)$, corresponding to a simple pole in eq. (11), due to the small- $k_{\perp}$ region. Note also the singularity of eq. (12) at $\gamma=1$, due to the behaviour $\hat{\sigma} \sim \rho \log (1 /$ $\rho)$ for $\rho \rightarrow 0$, or $\hat{\sigma}_{N} \sim\left(M^{2} / k_{\perp}^{2}\right) \log \left(k_{\perp}^{2} / M^{2}\right)$ for large $k_{\perp} \gg M$.

Let us now discuss the result of eq. (9) in various regimes, on the basis of the explicit form (12) of $h(\gamma)$. In our notation the small $\rho$ region corresponds to the moment index $N \rightarrow 0$, and the perturbative regime is defined by $\bar{\alpha}_{\mathrm{s}} / N \ll 1$ in moment space or by $\bar{\alpha}_{\mathrm{s}}$ $\times \log (1 / \rho) \ll \log \left(M^{2} / Q_{0}^{2}\right)$ in energy space. In this region, the $\gamma$-integral can be performed by going to the pole

$1=\frac{\bar{\alpha}_{\mathrm{s}}}{N} f(\gamma)$,

which implicitly defines the anomalous dimension $\gamma=\gamma\left(\bar{\alpha}_{s} / N\right)$, and by expanding eqs. (10b) and (12) in the form

$$
f(\gamma)=\frac{1}{\gamma}+\mathrm{O}\left(\hat{\gamma}^{2}\right)
$$


$h(\gamma)=h(0)\left[1+\frac{41}{21} \gamma+\mathrm{O}\left(\gamma^{2}\right)\right]$,

$h(0)=\frac{28}{9} \pi \alpha \alpha_{\mathrm{s}} e_{\mathrm{Q}}^{2}$.

At first non-trivial order, $\gamma \simeq \bar{\alpha}_{\mathrm{s}} / N$, and the photoproduction cross section is

$\sigma_{N}\left(\frac{M^{2}}{Q_{0}^{2}}\right)=\frac{h(0)}{4 M^{2}}\left(1+\frac{41 \bar{\alpha}_{\mathrm{s}}}{21 N}+\ldots\right)\left(\frac{M^{2}}{Q_{0}^{2}}\right)^{\alpha_{\mathrm{s}} / N}$,

$\sigma_{N}^{(1)}\left(\frac{M^{2}}{Q_{0}^{2}}\right)=\frac{h(0)}{4 M^{2}} \frac{\bar{\alpha}_{\mathrm{s}}}{N}\left(\frac{41}{21}+\log \frac{M^{2}}{Q_{0}^{2}}\right)$,

thus reproducing the lowest order correction of eq. (1), as expected. Further terms in the perturbative expansion are obtained from the power series in eq. (14) and from eq. (13), which defines the perturbative branch ${ }^{\# 2}$ of the anomalous dimension

$\gamma\left(\frac{\bar{\alpha}_{\mathrm{s}}}{N}\right)=\frac{\alpha_{\mathrm{s}}}{N}+2 \zeta(3)\left(\frac{\bar{\alpha}_{\mathrm{s}}}{N}\right)^{4}+\ldots$,

provided $N / \bar{\alpha}_{\mathrm{s}}>f\left(\gamma_{\min }\right)=4 \log 2$.

If $\bar{\alpha}_{\mathrm{s}} / N$ is too large or $\rho$ is too small $\left[\bar{\alpha}_{\mathrm{s}} \log (1 / \rho)>\log \left(M^{2} / Q_{0}^{2}\right)\right]$ the perturbative branch (16) is no longer meaningful, the solutions of eq. (13) being complex. In this case, it is more convenient to discuss directly the energy dependence of the cross section, which is obtained from eq. (9) by inverse Mellin transform. By performing the $N$-integration first at the pole in eq. (13), we obtain for $s \gg 4 M^{2}$

$$
\begin{aligned}
& 4 M^{2} \sigma\left(\rho, \frac{M^{2}}{Q_{0}^{2}}\right) \\
& \simeq \int_{1 / 2-\mathrm{i} \infty}^{1 / 2+\mathrm{i} \infty} \frac{\mathrm{d} \gamma}{2 \pi \mathrm{i}} \frac{\bar{\alpha}_{\mathrm{s}} f(\gamma)}{\gamma} h(\gamma)\left(\frac{M^{2}}{Q_{0}^{2}}\right)^{\gamma}\left(\frac{s}{4 M^{2}}\right)^{\bar{\alpha}_{\mathrm{s}} f(\gamma)} .
\end{aligned}
$$

The $\gamma$-integral is then evaluated by a saddle point method. The stationary phase condition is

$\bar{\alpha}_{s} L f^{\prime}(\bar{\gamma})+l=0 \quad\left(l=\log \frac{M^{2}}{Q_{0}^{2}}, L=\log \frac{1}{\rho}\right)$,

and yields the expression

\footnotetext{
\#2 $f(\gamma)$ has pole singularities at $\gamma=0$ and $\gamma=1$ and a minimum at $\gamma=\frac{1}{2}$. Thus there are [1] two real branches of $\gamma\left(\bar{\alpha}_{\mathrm{s}} / N\right)$ or none according to whether $N / \bar{\alpha}_{\mathrm{s}}>4 \log 2$ or $N / \bar{\alpha}_{\mathrm{s}}<4 \log 2$.
}

$$
\begin{aligned}
& 4 M^{2} \sigma\left(\rho, \frac{M^{2}}{Q_{0}^{2}}\right) \\
& \simeq\left(\frac{M^{2}}{Q_{0}^{2}}\right)^{\bar{\gamma}}\left(\frac{s}{4 M^{2}}\right)^{\bar{\alpha} f(\bar{\gamma})} \frac{\bar{\alpha}_{\mathrm{s}} f(\bar{\gamma})}{\bar{\gamma}} \frac{h(\bar{\gamma})}{\left[2 \pi \bar{\alpha}_{\mathrm{s}} L f^{\prime \prime}(\bar{\gamma})\right]^{1 / 2}} .
\end{aligned}
$$

At intermediate energies, such that $\bar{\alpha}_{\mathrm{s}} L \ll l$, the stationary point $\bar{\gamma} \simeq\left(\bar{\alpha}_{\mathrm{s}} L / l\right)^{1 / 2}$, and the cross section behaviour is

$$
\begin{gathered}
4 M^{2} \sigma\left(\rho, \frac{M^{2}}{Q_{0}^{2}}\right) \simeq \frac{1}{\sqrt{4 \pi}}\left(\bar{\alpha}_{\mathrm{s}} l\right)^{1 / 4} L^{-3 / 4} h(0) \\
\times\left[1+\frac{41}{21}\left(\frac{\bar{\alpha}_{\mathrm{s}} L}{l}\right)^{1 / 2}\right] \exp \left(2 \sqrt{\bar{\alpha}_{s} L l}\right) .
\end{gathered}
$$

We recognize here the exponential behaviour of Lipatov's structure function in the one-loop regime $\left(\gamma \simeq \bar{\alpha}_{\mathrm{s}} / N\right)$, while $h(\gamma)$ takes its perturbative form in eq. (14), as discussed before.

In the opposite regime of extreme energies $\left(\bar{\alpha}_{\mathrm{s}} L \gg l\right)$, the saddle point drifts towards the minimum of $f(\gamma)$ at $\gamma=\frac{1}{2}$, so that we obtain, approximately,

$$
\begin{aligned}
& 4 M^{2} \sigma\left(\rho, \frac{M^{2}}{Q_{0}^{2}}\right) \\
& \simeq \sqrt{\frac{M^{2}}{Q_{0}^{2}}}\left(\frac{s}{4 M^{2}}\right)^{4 \bar{\alpha}_{s} \log 2} \frac{8 \bar{\alpha}_{s} \log 2}{\left[56 \pi \zeta(3) \bar{\alpha}_{s} L\right]^{1 / 2}} h\left(\frac{1}{2}\right) .
\end{aligned}
$$

where, by eq. (12),

$$
h\left(\frac{1}{2}\right)=\frac{3 \pi^{3}}{2} \alpha \alpha_{\mathrm{s}} e_{\mathrm{Q}}^{2} \text {. }
$$

We see in eq. (21) the power dependence on $s$ of the "perturbative pomeron".

The value $h\left(\frac{1}{2}\right)$ on the RHS of eq. (21a) represents the dynamical part of the coefficient factor. It is the result of the resummation of the large perturbative contributions $\left[\alpha_{\mathrm{s}} \log (1 / \rho)\right]^{n}$. Indeed, in a naive renormalization group approach (i.e. approximating $\hat{\sigma}\left(\rho / z, k_{\perp}^{2} / 4 M^{2}\right)$ with its $k_{\perp}^{2} \ll M^{2}$ limit in the factorization formula (3)) it would be replaced by $h(0)$ in eq. (21a). By comparing eqs. (21b) and (14b) we see that $h\left(\frac{1}{2}\right)$ is approximately a factor of five larger than $h(0)$, leading to a corresponding in- 
crease in the photoproduction total cross section at very high energies.

We want to emphasize some other interesting features of the coefficient resummation just obtained in eqs. (19) and (21). First, one may wonder why this resummation is dependent on such detailed integrals as in eq. (11), leading to the result (12). This is because, in the $k_{\perp}$ integration of eq. (9), only the small $k_{\perp} / M$ region is universal (due to the factorization of collinear singularities) and determines the value of $h(0)$ in eq. (11), as noticed before. The intermediate values of $k_{\perp}=\mathrm{O}(M)$, contributing to the coefficient factors, are instead dependent on the shape of $\hat{\sigma}\left(\rho, k^{2} / 4 M^{2}\right)$, which does not provide a sharp determination of $k_{\perp} / M$.

The second point to be understood is why the resummed asymptotic value in eq. (21b) is just a finite constant, showing no trace of the leading powers of $\log (1 / \rho)$ it originated from. This is due to the smoothness of $h(\gamma)$ for $0 \leqslant \gamma<1$, which in turn derives from the fact that $\hat{\sigma}\left(\rho, k^{2} / 4 M^{2}\right)$ is uniformly small, of order $\rho$, even close to the phase space boundary of large $k_{\perp}=\mathrm{O}(\sqrt{s})$. Hence, $h(\gamma)$ is not sensitive to the widening of the $k_{1}$ phase space. In contrast, a point-like source would yield, typically,

$$
\begin{aligned}
& \hat{\sigma}_{\text {point }}(\rho)=\frac{1}{\rho} \delta\left(\frac{1}{\rho}-1-\frac{k_{\perp}^{2}}{Q^{2}}\right), \\
& \hat{\sigma}_{N, \text { point }}=\left(1+\frac{k_{\perp}^{2}}{Q^{2}}\right)^{-N} \stackrel{N \rightarrow 0}{\longrightarrow} 1,
\end{aligned}
$$

thus causing an additional singularity of eq. (11) at $\gamma=N$, and eventually double-log terms $\left(\sim \alpha_{\mathrm{s}} / N^{2}\right)$ in the coefficient function.

The above remarks illustrate the fact that the QQ system is a non-local probe of the gluon $k_{\perp}$-dependence, which cuts off the $k_{1}$-integration around $k_{\perp} \simeq M \ll \sqrt{s}$. For a local probe large values of $k_{\perp}=$ $\mathrm{O}(\sqrt{s})$ are allowed, yielding a much stronger singularity of the coefficient of type $\alpha_{\mathrm{s}} / N^{2}$ (as for timelike jet evolution [1]). It is rather peculiar that no such local probe seems available in nature for gluons. Indeed, even produced jets, corresponding to emitted gluons, are screened by strong virtual corrections $[8,9]$, which do not allow values of $k_{\perp}$ larger than the jet mass. This fact seems to be yet another manifestation of colour confinement.

Our treatment of the coefficient factor can be ex- tended to other heavy flavour production processes as well as to Higgs and vector boson production. In particular, for the heavy flavour hadroproduction case (fig. 3), where similar perturbative features have been computed $[2,5]$, the $k_{\perp}$-factorization due to Regge behaviour involves the hard subprocess $\mathrm{g}\left(k_{1}\right)+$ $\mathrm{g}\left(k_{2}\right) \rightarrow \mathrm{Q}+\overline{\mathrm{Q}}$, with one gluon per incoming hadron. The form of the heavy flavour cross section that we propose is again of convolution type, i.e.,

$$
\begin{aligned}
& 4 M^{2} \sigma\left(\rho, M^{2}\right) \\
& =\int \mathrm{d}^{2} \boldsymbol{k}_{1} \mathrm{~d}^{2} \boldsymbol{k}_{2} \int_{0}^{1} \frac{\mathrm{d} z_{1}}{z_{1}} \frac{\mathrm{d} z_{2}}{z_{2}} \hat{\sigma}\left(\frac{\rho}{z_{1} z_{2}}, \boldsymbol{k}_{1}, \boldsymbol{k}_{2} ; M^{2}\right) \\
& \quad \times \mathscr{F}\left(z_{1}, \boldsymbol{k}_{1}\right) \mathscr{F}\left(z_{2}, \boldsymbol{k}_{2}\right) .
\end{aligned}
$$

where the off-shell Born cross section $\hat{\sigma}$ is again defined by coupling the incoming gluons to external partons with eikonal vertices. So far, this quantity is known only for $\boldsymbol{k}_{1}^{2} \ll \boldsymbol{k}_{2}^{2}[2,5]$, which is only sufficient to discuss a few perturbative terms in eq. (23). Thus, a complete evaluation of the $k_{\perp}$-dependence, including the non abelian terms, is needed [7].

We would like to thank Luca Trentadue and Giuseppe Marchesini for interesting discussions and Jan Jenkins for typing the manuscript and drawing the figures.

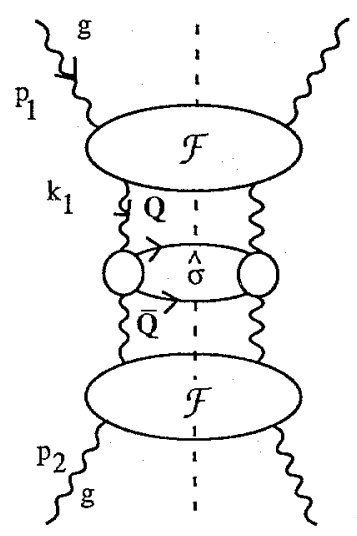

Fig. 3. Factorized structure of the heavy flavour hadroproduction cross section in the high energy limit. 


\section{References}

[1] A. Bassetto, M. Ciafaloni and G. Marchesini, Phys. Rep. 100 (1983) 201.

[2] P. Nason, S. Dawson and R.K. Ellis, Nucl. Phys. B 303 (1988) 607.

[3] R.K. Ellis and P. Nasoson, Nucl. Phys. B 312 (1989) 551.

[4] G. Altarelli, M. Diemoz, G. Martinelli and P. Nason, Nucl. Phys. B 308 (1988) 724.

[5] W. Beenakker, H. Kuijf, W.L. van Neerven and J. Smith, Phys. Rev. D 40 (1989) 54.
[6] L.N. Lipatov, Sov. J. Nucl. Phys. 23 (1976) 338;

E.A. Kuraev, L.N. Lipatov and V.S. Fadin, Sov. Phys. JETP 45 (1977) 199;

Ya. Balitskii and L.N. Lipatov, Sov. J. Nucl. Phys. 28 (1978) 6.

[7] S. Catani, M. Ciafaloni and F. Hautmann, in preparation.

[8] M. Ciafaloni, Nucl. Phys. B 296 (1987) 249.

[9] S. Catani, F. Fiorani and G. Marchesini, Nucl. Phys. B 336 (1990) 18. 